\title{
DDT and pyrethroid resistance in Anopheles arabiensis from South Africa
}

\author{
Luisa Nardini ${ }^{1,2}$, Riann N Christian ${ }^{1,2}$, Nanette Coetzer ${ }^{3}$ and Lizette L Koekemoer ${ }^{1,2^{*}}$
}

\begin{abstract}
Background: Pyrethroid resistance has been well documented in Anopheles arabiensis, one of the major African malaria vectors, and the predominant malaria vector in South Africa.

Methods: In this study, the genetic basis of pyrethroid resistance in a selected laboratory strain of An. arabiensis from South Africa was investigated using a custom-made microarray, known as the An. gambiae detoxification chip.

Results: A large number of P450 genes were over-transcribed, as well as a suite of redox genes and glutathione $\mathrm{S}$-transferases. The five genes that showed the highest level of gene transcription when compared with an insecticide susceptible strain were: CYP6AG2, CYPZ1, TPX2, CYPZ2 and CYP6P1.

Conclusions: Permethrin resistance in South African An. arabiensis is associated with increased transcription of multiple genes, and a large proportion of these genes were also previously recorded as over-transcribed in another An. arabiensis strain selected for resistance to DDT with cross-resistance to deltamethrin. The deltamethrin resistance developed de novo in the DDT-selected strain and is most likely due to increased transcription of those genes associated with DDT resistance. However, of particular interest was the fact that the strain selected for resistance to pyrethroids did not develop de novo resistance to DDT. These differences are compared and discussed.
\end{abstract}

Keywords: South Africa, Insecticide resistance, Cytochrome P450, Glutathione S-transferase, Permethrin, Deltamethrin, Microarrays

\section{Background}

Pyrethroids are the most commonly used insecticides for malaria vector control. They are most often used for indoor residual spraying, and are the only class of insecticide approved for treatment of bednets due to their low levels of mammalian toxicity [1-3]. However, pyrethroid resistance in African malaria vectors, including Anopheles arabiensis, is well documented and has been reported in many African countries.

The Global Plan for Insecticide Resistance Management in Malaria Vectors (GPIRM) [1] provides a generic guideline for managing insecticide resistance where it occurs. Strategies for preventing and managing insecticide

\footnotetext{
* Correspondence: lizettek@nicd.ac.za

'Division of the National Health Laboratory Services, Vector Control Reference Laboratory, Centre for Opportunistic, Tropical and Hospital Infections, National Institute for Communicable Diseases, Private Bag X4, Johannesburg 2131 Sandringham, South Africa

${ }^{2}$ WITS Research Institute for Malaria, School of Pathology, Faculty of Health Sciences, University of the Witwatersrand, Johannesburg, South Africa
} Full list of author information is available at the end of the article resistance are imperative to ensure that the limited number of insecticides available for vector control are protected. As part of this strategy, insecticide resistance should be monitored through routine surveillance and the mechanisms of insecticide resistance should be characterised where possible [1]. Information on insecticide resistance mechanisms in malaria vectors can be obtained using several techniques that vary in sophistication and cost. The more costly methodologies are not feasible in many African countries where the disease burden is the greatest.

Resistance to pyrethroids is mainly due to two mechanisms; enhanced enzyme detoxification or reduced target site sensitivity. Cytochrome oxidases (P450s), glutathione $\mathrm{S}$-transferases (GSTs) and esterases are generally associated with enzymatic detoxification of insecticides, while mutations in the voltage-gated sodium channel (commonly referred to as knockdown resistance or " $k d r$ ") effectively decrease target site sensitivity $[4,5]$. The occurrence of $k d r$

\section{Biomed Central}


mutations is easily detected through the use of a polymerase chain reaction (PCR) assay [5] but this mechanism is not clearly correlated with all instances of pyrethroid resistance, especially deltamethrin resistance [6]. Identifying metabolic based resistance is more challenging and costly, especially when using microarrays [7]. The use of microarray technology is particularly helpful as it allows for rapid analysis of numerous genes simultaneously, and as such provides substantial genetic information relating to a particular phenotype.

Genes associated with pyrethroid resistance include the esterase, GST and P450 super families [8-13]. Metabolic detoxification of pyrethroids has been demonstrated in An. gambiae [12,14,15], An. arabiensis $[8,10,16]$ and An. funestus (reviewed by [17]). Anopheles arabiensis is a major vector of malaria in Africa including South Africa $[18,19]$. Historically, this species was considered to be susceptible to all classes of insecticide. In 1996 an extensive survey of insecticide susceptibility in An. arabiensis was conducted in three areas in South Africa (KwaZulu-Natal [KZN], Limpopo Province and the Kruger National Park) [20]. Bioassays revealed that samples of An. arabiensis from all three areas were susceptible to DDT and deltamethrin [20].

Current malaria vector control in South Africa is based on a mosaic approach using DDT and pyrethroids [19]. These interventions are the mainstay of mosquito vector control in South Africa even though An. arabiensis is both exophilic and endophilic. In 2003, An. arabiensis sampled from two areas in KZN were found to be resistant to DDT, but susceptible to deltamethrin [21].

Subsequent studies were initiated to investigate the molecular basis for the reported DDT resistance in KZN An. arabiensis. The use of laboratory strains to study resistance mechanisms carries the advantage of excluding factors that can confound the analysis (e.g. exposure to agricultural insecticides, effect of temperature, larval diet, etc). Laboratory based DDT selection pressure was exerted on this population over a period of several years and resulted in the fixation of the L1014F $k d r$ mutation [8]. In addition to target site resistance, metabolic detoxification was also identified and numerous gene transcripts were over-expressed and associated with the resistant phenotype [8].

Three years after the discovery of DDT resistance, pyrethroid resistance was also reported in wild caught $A n$. arabiensis from KZN [16]. This population was colonised in 2005 and selected for resistance to permethrin in order to characterise the resistance mechanisms involved. Pyrethroid resistance in this population (named KWAG-perm) was primarily associated with increased activity of monooxygenases (P450's) [16]. Analysis of six specific P450 genes showed increased transcription for three genes in association with the pyrethroid resistant phenotype [22].
The aim of this study was to evaluate the levels of transcription of detoxification enzymes associated with the South African permethrin resistant strain (KWAGperm) using a high throughput microarray analysis to supplement the information obtained by Munhenga and Koekemoer [22]. In addition to this, the transcript profiles between the pyrethroid-selected (KWAG-perm, permethrin resistant) and DDT-selected (MBN-DDT, DDT and pyrethroid resistance) strains were compared to identify both strain specific transcripts as well as transcripts shared between these two strains. The implications of these results for malaria control are discussed.

\section{Methods \\ Mosquito strains \\ Pyrethroid resistant strain (KWAG-perm)}

The An. arabiensis permethrin resistant strain, KWAGperm, and the equivalent susceptible strain, KWAG-base, were used for this experiment. KWAG-base originates from Mamfene in KwaZulu-Natal (KZN) province, South Africa. This population was characterised as permethrin resistant at the time of colonisation [16]. However, in the absence of selection by permethrin exposure, the laboratory strain reverted back to being susceptible (after a period of one year). When permethrin selection was reintroduced, resistance rapidly returned (within four generations) and this strain was named KWAG-perm (Table 1) [16]. Mosquitoes were reared under standard insectary conditions $\left(26^{\circ} \mathrm{C} \pm 2^{\circ} \mathrm{C}, 70-80 \%\right.$ relative humidity, and 12:12 light:dark cycle).

\section{$D D T$ resistant strain (MBN-DDT)}

Details on this strain can be found in Nardini et al. [8]. Briefly, MBN-base was colonised from Mamfene, KZN, and a sub-strain was selected for resistance to DDT. The selected strain, MBN-DDT, is resistant to DDT ( $0 \%$ mortality) as well as permethrin (4\%), deltamethrin (34\%), bendiocarb (77.5\%) and propoxur (65.3\%) through crossresistance mechanisms (Table 1). The strain is homozygous for the L1014F $k d r$ mutation which is mainly responsible for the observed DDT and permethrin resistance [8]. The deltamethrin resistance is mainly due to enhanced monooxygenase enzymatic detoxification [8].

Table 1 Summary of the An. arabiensis strains used in the study

\begin{tabular}{lll}
\hline Strain & Selected on: & Resistant to: \\
\hline KWAG-base & No selection & - \\
KWAG-perm & Permethrin (0.75\%) & Permethrin \\
MBN-base & No selection & DDT (low-level) \\
MBN-DDT & DDT (4\%) & $\begin{array}{l}\text { DDT, deltamethrin and permethrin } \\
\text { (cross-resistance) }\end{array}$ \\
\hline
\end{tabular}




\section{RNA extractions and preparation of amplified mRNA Pyrethroid resistant strain (KWAG-perm)}

RNA extractions were carried out according to Nardini $e t$ al. [8]. Briefly, female mosquitoes were maintained on a $10 \%$ sugar solution and RNA was extracted from 15 mosquitoes three days after emergence, representing one biological repeat. RNA was extracted for three biological repeats using the PicoPure ${ }^{ø}$ RNA Isolation Kit (Arcturus) according to supplied methodology (with the inclusion of a DNase treatment, as described in the user manual). RNA was quantified using a NanoDrop and quality was assessed using an Agilent 2100 Bioanalyzer. Extracted RNA was stored at $-70^{\circ} \mathrm{C}$. In order to obtain larger quantities of mRNA for microarrays, the RiboAmp ${ }^{\text {тм }}$ RNA Amplification Kit (Arcturus) was used. Amplified mRNA was quantified using a NanoDrop.

\section{Preparation of microarrays and data analyses Pyrethroid resistant strain (KWAG-perm)}

Three independent biological repeats were used, and for each biological repeat, two technical repeats were performed that included dye swaps in order to compensate for dye bias. Microarrays were prepared as described in Nardini et al. [8].

The arrays were scanned using the Genepix 4000B scanner (Molecular Devices, USA). The photomultiplier tube (PMT) values were adjusted to give a pixel ratio of approximately 1 . Spot quality and background intensities were examined and corrected using Genepix Pro 6.0 software (Axon Instruments, USA). Saturated features were excluded from the analysis.

Gene expression data were analysed using Limma version 2.12.0 (Bioconductor) [23] in $\mathrm{R}$, version 2.8.0 (http://cran.r-project.org/bin/windows/base/old/2.8.0/). Raw intensity values for each spot were calculated, and then background corrected by the method "normexp" with an offset of 50 [24]. The corrected intensity values were transformed to log-ratios and then normalised. Composite Loess was used for within array normalisation. In this method, control spots and features, per sub-array, are used for producing non-linear, best-fit lines [25]. The use of control spots ensures that the resulting best-fit line is not biased by differential expression of genes. Conversely, the use of all genes for normalisation improves stability with respect to the number of spots and, most importantly, provides flexibility in terms of print-tip group trends that might be observed where sub-array Loess curves are used [26]. Print-tip peculiarities were present in some slides, hence the choice of the normalisation method. The "Aquantile" method was used for between array normalisation. Genes with adjusted $p$-values $\leq 0.05$ and fold-changes (FCs) $\geq 2.0$ were considered to be statistically significant. These data have been deposited into Vectorbase (https://www.vectorbase.org).

\section{Quantitative real-time PCR (qPCR) Pyrethroid resistant strain (KWAG-perm)}

Real-time PCR was carried out in order to validate the outcome of the microarray experiments. RNA was extracted from 15 three day old An. arabiensis females (represents one biological repeat, and three biological repeats were prepared) that had been supplied with $10 \%$ sugar solution. TRI-Reagent ${ }^{\oplus}$ solution (Sigma-Aldrich) was used according to the supplied methodology with a DNase treatment included. Samples were reverse-transcribed into cDNA using the QuantiTect ${ }^{\oplus}$ Reverse Transcription Kit (Qiagen). The cDNA was stored at $-70^{\circ} \mathrm{C}$ until required for qPCR.

Two genes, CYP6AG2 and TPX2, were evaluated using real-time PCR. Beacon Designer ${ }^{\text {TM }}$ (Premier Biosoft) or Invitrogen's free online primer design tool, OligoPerfect ${ }^{\mathrm{TM}}$ Designer, were used to design primers. These were based on An. gambiae sequence information (CYP6AG2: GB AY745225; TPX2: TIGR TC48596). The reference gene used for qPCR was based on that of Munhenga and Koekemoer [22], where studies were conducted in the laboratory on the same mosquito strains. These authors reported that $18 S$ rRNA showed the most stable expression of the six potential reference genes tested. The forward and reverse primer sequences for $18 S$ were the same as those of Munhenga and Koekemoer [22]. Each PCR reaction comprised $12.5 \mu \mathrm{IQ}^{\mathrm{Tm}}$ SYBR super-mix (Bio-Rad), $4 \mu \mathrm{l}$ primer (primer concentration optimised for each primer set), $1 \mu \mathrm{l} \mathrm{cDNA} \mathrm{(100} \mathrm{ng/ \mu l)} \mathrm{and} \mathrm{nuclease-}$ free water to a final volume of $25 \mu \mathrm{l}$. Cycling conditions were as follows: $93^{\circ} \mathrm{C}$ for 3 minutes, followed by 35 cycles of $\left(94^{\circ} \mathrm{C} / 20\right.$ seconds, $\times{ }^{\circ} \mathrm{C} / 25$ seconds, $72^{\circ} \mathrm{C} / 30$ seconds), and a final extension at $72^{\circ} \mathrm{C}$ for 10 minutes (followed by melt curve analysis). Primer sequences and annealing temperatures are described in Table 2. Data were analysed using the Pfaffl [27] method. For each gene of interest, the PCR product was sequenced in both directions, so that the presence of the correct product was confirmed (in addition to melt-curve analysis). Where necessary (for small amplicons i.e. < 110 base pairs), samples were cloned and then sequenced.

\section{Results}

KWAG-base and KWAG-perm have been evaluated for insecticide resistance on an ongoing basis in the laboratory. The base strain showed very little resistance to permethrin $(97.8 \%$ mean mortality 24 hours post exposure to $0.75 \%$ permethrin), while the resistant strain showed a high frequency of survivors on permethrin (42\% mean mortality 24 hours post exposure to $0.75 \%$ permethrin) [22].

Microarray experiments indicated that 29 genes were over-transcribed according to the criteria outlined above. Most of these were $\mathrm{P} 450$ genes (55\%), followed by redox 


\begin{tabular}{|c|c|c|c|}
\hline Gene & Primer sequence & $\begin{array}{l}\text { Primer } \\
\text { concentration }\end{array}$ & $\begin{array}{l}\text { Annealing } \\
\text { temperature }\end{array}$ \\
\hline \multirow[t]{2}{*}{ CYPGAG2 } & $\begin{array}{l}\text { F 5'- TTG TGC TGC CGT } \\
\text { ACT ATT CG-3' }\end{array}$ & $2.0 \mu \mathrm{M}$ & $59.4^{\circ} \mathrm{C}$ \\
\hline & $\begin{array}{l}\text { R 5'- TAC TAT CGC CCG } \\
\text { TCT CAC CT - -3' }\end{array}$ & & \\
\hline \multirow[t]{2}{*}{ TPX2 } & $\begin{array}{l}\text { F 5'- GGA TGT TTG TGG } \\
\text { GGA ATA CG -3' }\end{array}$ & $3.5 \mu \mathrm{M}$ & $56.3^{\circ} \mathrm{C}$ \\
\hline & $\begin{array}{l}\text { R 5'- TGT GCG ATT AGC } \\
\text { CTC CTC TT-3' }\end{array}$ & & \\
\hline \multirow[t]{2}{*}{185} & $\begin{array}{l}\text { F 5'- TAC CTG GGC GTT } \\
\text { CTA CTC -3' }\end{array}$ & a & b \\
\hline & $\begin{array}{l}\text { R 5'- CTT TGA GCA CTC } \\
\text { TAA TाT GTT C -3' }\end{array}$ & & \\
\hline
\end{tabular}

${ }^{\mathrm{a}}$ Primer concentration was the same as that of CYP6AG2 and TPX4 when used as a reference gene in each case.

${ }^{\mathrm{b}}$ Annealing temperature was the same as that of CYP6AG2 and TPX4 when used as a reference gene for each.

genes (21\%), and GSTs (14\%). A group of additional genes, such as cytochrome c, a ribosomal gene (RPS26) and a receptor protein (GPR npy 3) accounted for $10 \%$ of the over-transcribed genes. The four genes with the highest transcript abundance were CYP6AG2, CYP6Z1, TPX2 and CYP6Z2 (Table 3), in order of statistical significance (i.e. adjusted $p$-value).

Two significantly over-transcribed genes, CYP6AG2 and $T P X 2$, were selected for $\mathrm{qPCR}$ validation of microarray data. The two other "top" genes (CYP6Z1 and CYP6Z2) were already evaluated with qPCR [22]. CYP6AG2 was the most significantly over-transcribed gene, and TPX2 is of interest because the TPX genes have been found to be important in other studies reporting on insecticide resistance $[8,10]$. The genes CYP6AG2 and TPX2 produced fold change values of 2.9 and 4.5 respectively (Figure 1 ). The FC value of CYP6AG2 and TPX2 were comparable to those obtained by microarray analysis.

Munhenga and Koekemoer [22] used qPCR to evaluate transcription of specific genes (identified through published literature) that have been implicated in permethrin resistance in $A n$. arabiensis. These included $C Y P 6 Z 1$, CYP6Z2, CYP6Z3, CYP6M2, CYP6P3 and CYP4G16. Of these, five appeared on the list of over-transcribed genes in this microarray study (Table 3 ) and three of these genes [CYP6Z1 (4.7-fold), CYP6Z2 (1.7-fold) and CYP6M2 (2.2-fold)] were significantly over-transcribed in the qPCR study [22]. The FC values of all six genes analysed by Munhenga and Koekemoer [22] were compared with the FC values obtained here for the same genes using microarray analysis (Figure 2). The FC values obtained by microarray analyses were compared with those obtained using $\mathrm{qPCR}$ for each gene using a $t$-test. The FC values of only three of the genes were significantly different to the FC values obtained by microarray analysis. These were CYP6Z2 $(p=0.0251)$, CYP6Z3 $(p=0.0219)$ and CYP6M2 $(p=0.0092)$.

\section{Transcript profile comparison between the DDT-selected} strain and the pyrethroid-selected strain (this study)

Both MBN-DDT and KWAG-perm originate from Mamfene $(\mathrm{KZN})$. However, they were placed under different insecticide selection pressures (depending on the insecticide resistance phenotypes present at the time of colonisation). The list of over-transcribed probes obtained in the permethrin-resistant strain (KWAG-perm, pyrethroid resistance only) was compared with that obtained in the DDT selected strain (MBN-DDT, pyrethroid and DDT resistance [8]) (Figure 3). In MBN-DDT, $k d r$ was closely associated with DDT and permethrin resistance, while deltamethrin resistance was largely based on enhanced enzymatic detoxification. Five GST transcripts were unique in the DDT-selected strain (MBN-DDT), while 14 transcripts were unique to the permethrin-selected strain (KWAG-perm). Fifteen gene transcripts were shared between these of which 10 (66\%) belonged to the P450-enzyme family.

\section{Discussion}

The use of controlled insecticide selection in the laboratory aids in the study of changes in resistance status and its effect on certain adaptive features [28]. A list of 29 over-transcribed genes according to the criteria used (adjusted $p$-value $\leq 0.05 ; \mathrm{FC} \geq 2$ ) was produced for the KWAG-perm strain. Most of the genes that were overtranscribed belong to the cytochrome P450 superfamily, supporting the findings of previous studies [16,22]. The four most statistically significant $\mathrm{P} 450$ genes included CYP6AG2, CYP6Z1, CYP6Z2 and CYP6P1, all of which belong to the CYP6 family and are well-known for their role in insecticide resistance $[14,29]$. The most significant gene, CYP6AG2, is not well characterised and it would be valuable to determine the functionality of this enzyme. This gene is located on the chromosome arm $2 \mathrm{R}$, a region that is not typically linked to pyrethroid resistance. Another gene in this family, CYP6AG1, was identified in a wild deltamethrin resistant $A n$. arabiensis population from Cameroon [10]. CYP6Z1, CYP6Z2 and CYP6M2 showed increased transcription in this study as well as in the study by Munhenga and Koekemoer [22].

Pyrethroids are also known to cause oxidative stress by inducing lipid peroxidation, protein oxidation, and depletion of reduced glutathione [30]. This effectively increases the toxicity of the insecticide. A system of enzymes, including the superoxide dismutases (SODs), catalases, peroxidases, cytochrome C and GSTs, is present and provides defense against these reactive oxygen species (ROS) $[31,32]$. If ROS are not metabolised, they damage 
Table 3 List of probes over-transcribed in the resistant phenotype, KWAG-perm

\begin{tabular}{|c|c|c|c|c|c|}
\hline Gene & Function & Adj. $p$-value & FC & Accession number & Location \\
\hline CYP6AG2 & Cytochrome P450 & $2.58 \mathrm{E}-7$ & 4.1 & AY745224 & $2 R$ \\
\hline${ }^{\#}$ CYP6Z1 (oligo) & Cytochrome P450 & $3.05 \mathrm{E}-7$ & 4.7 & AF487535 & $3 R$ \\
\hline TPX2 & Thioredoxin peroxidase & 4.56E-7 & 2.3 & TIGR: TC48596 & $3 L$ \\
\hline${ }^{\#}$ CYP6Z2 & Cytochrome P450 & $1.14 \mathrm{E}-6$ & 3.6 & XM_317252 & $3 R$ \\
\hline CYP6P1 & Cytochrome P450 & $1.14 \mathrm{E}-6$ & 2.2 & AY028785 & $2 \mathrm{R}$ \\
\hline GSTU1 & Glutathione S-transferase & $3.03 \mathrm{E}-6$ & 2.2 & XM_309135 & $x$ \\
\hline SOD2 & Superoxide dismutase & $3.81 \mathrm{E}-6$ & 3.5 & AY524130 & $2 \mathrm{~L}$ \\
\hline CYP12F2 & Cytochrome P450 & $3.81 \mathrm{E}-6$ & 2.9 & AY176050 & $3 R$ \\
\hline CYPGY2 & Cytochrome P450 & $3.81 \mathrm{E}-6$ & 2.2 & AY193728 & $3 R$ \\
\hline \multirow[t]{2}{*}{ GPR npy 3} & \multirow[t]{2}{*}{ G protein coupled receptor } & \multirow[t]{2}{*}{ 4.83E-6 } & \multirow[t]{2}{*}{3.2} & ENSANG: & \multirow[t]{2}{*}{$2 \mathrm{R}$} \\
\hline & & & & G00000009317 & \\
\hline CYP9J5 & Cytochrome P450 & $9.14 \mathrm{E}-6$ & 3.2 & AY748830 & $3 L$ \\
\hline GPX1 & Glutathione peroxidase & $9.14 \mathrm{E}-6$ & 2.4 & AY842257 & $2 \mathrm{R}$ \\
\hline${ }^{\# C Y P 6 P 3 ~}$ & Cytochrome P450 & $1.68 \mathrm{E}-5$ & 2.6 & AF487534 & $2 R$ \\
\hline${ }^{\#}$ CYP6Z1 (CDNA) & Cytochrome P450 & $1.96 \mathrm{E}-5$ & 3.0 & AF487535 & $3 R$ \\
\hline CYP6M3 & Cytochrome P450 & $1.99 \mathrm{E}-5$ & 4.5 & AY193730 & $3 R$ \\
\hline SOD1 & Superoxide dismutase & $2.35 E-5$ & 2.7 & AY505417 & $3 L$ \\
\hline \#CYP6Z3 & Cytochrome P450 & $2.95 E-5$ & 3.0 & AY193727 & $3 R$ \\
\hline CYP6AK1 & Cytochrome P450 & $5.42 \mathrm{E}-5$ & 3.6 & AY745227 & $3 L$ \\
\hline CYP9M1 & Cytochrome P450 & 1.03E-4 & 2.6 & AY748836 & $3 R$ \\
\hline CYP12F4 & Cytochrome P450 & $1.68 \mathrm{E}-4$ & 2.3 & AY176048 & $3 R$ \\
\hline CYP4H24 & Cytochrome P450 & 2.05E-4 & 2.6 & AY062206 & $x$ \\
\hline${ }^{\# C Y P G M 2}$ & Cytochrome P450 & $3.38 \mathrm{E}-4$ & 4.6 & AY193729 & $3 R$ \\
\hline GSTD1-3 & Glutathione S-transferase & $6.83 \mathrm{E}-4$ & 2.0 & AF071163 & $2 \mathrm{R}$ \\
\hline CYP6M1 & Cytochrome P450 & 1.16E-3 & 2.2 & AY062208 & $3 R$ \\
\hline GSTS1-1 & Glutathione S-transferase & $1.64 \mathrm{E}-3$ & 2.5 & L07880 & $3 L$ \\
\hline \multirow[t]{2}{*}{ RPS26 } & \multirow[t]{2}{*}{ Ribosomal protein } & \multirow[t]{2}{*}{$2.16 \mathrm{E}-3$} & \multirow[t]{2}{*}{3.1} & EMBL: & \multirow[t]{2}{*}{$3 L$} \\
\hline & & & & 4A3A-AAM-G-11-R & \\
\hline GPX3 & Glutathione peroxidase & 2.16E-3 & 2.1 & AY745228 & $x$ \\
\hline TPX4 & Thioredoxin-dependent peroxidase & $3.60 \mathrm{E}-3$ & 2.8 & AY745235 & $3 \mathrm{~L}$ \\
\hline GSTS1-2 & Glutathione S-transferase & 4.15E-3 & 2.3 & AF513639 & $3 L$ \\
\hline Cytochrome_C & Cytochrome c & $2.17 \mathrm{E}-2$ & 2.0 & TIGR: TC48590 & $3 R$ \\
\hline
\end{tabular}

Probes are listed in order of significance (adjusted [adj.] $p$-value), and then by fold change value. The accession number refers to Genbank, unless otherwise specified in the table; FC Fold change, $E$ Exponent.

\#Gene transcript validated by qPCR by Munhenga and Koekemoer [22].

important compounds such as lipids, proteins, nucleic acids and carbohydrates, and ultimately cause cell death [32]. A number of redox enzymes showed elevated levels of transcription in KWAG-perm, which may be associated with resistance to pyrethroids. These included thioredoxin peroxidases (TPX2 and TPX4), glutathione peroxidases (GPX1 and GPX3), superoxide dismutases (SOD1 and $S O D 2$ ) and cytochrome C. Superoxide radicals are converted to hydrogen peroxide and oxygen by SODs, and hydrogen peroxide is converted to water and oxygen by catalases; or to water, by peroxidases [33].
Four GSTs (GSTU1, GSTD1-3, GSTS1-1, GSTS1-2) were also over-transcribed in KWAG-perm. Overtranscription of GSTs has been observed before in pyrethroid resistant An. arabiensis [10], An. gambiae [7,9,34] and in other insects [35-37]. In conjunction with glutathione, GSTs function as antioxidants by limiting peroxidation and by limiting (termination) "free-radical cascades" (see [30]). In addition, the GSTs are able to bind to pyrethroids and provide protection by sequestration [37].

In the recent study of Nardini et al. [8], the authors identified genes that were over-transcribed in a DDT 


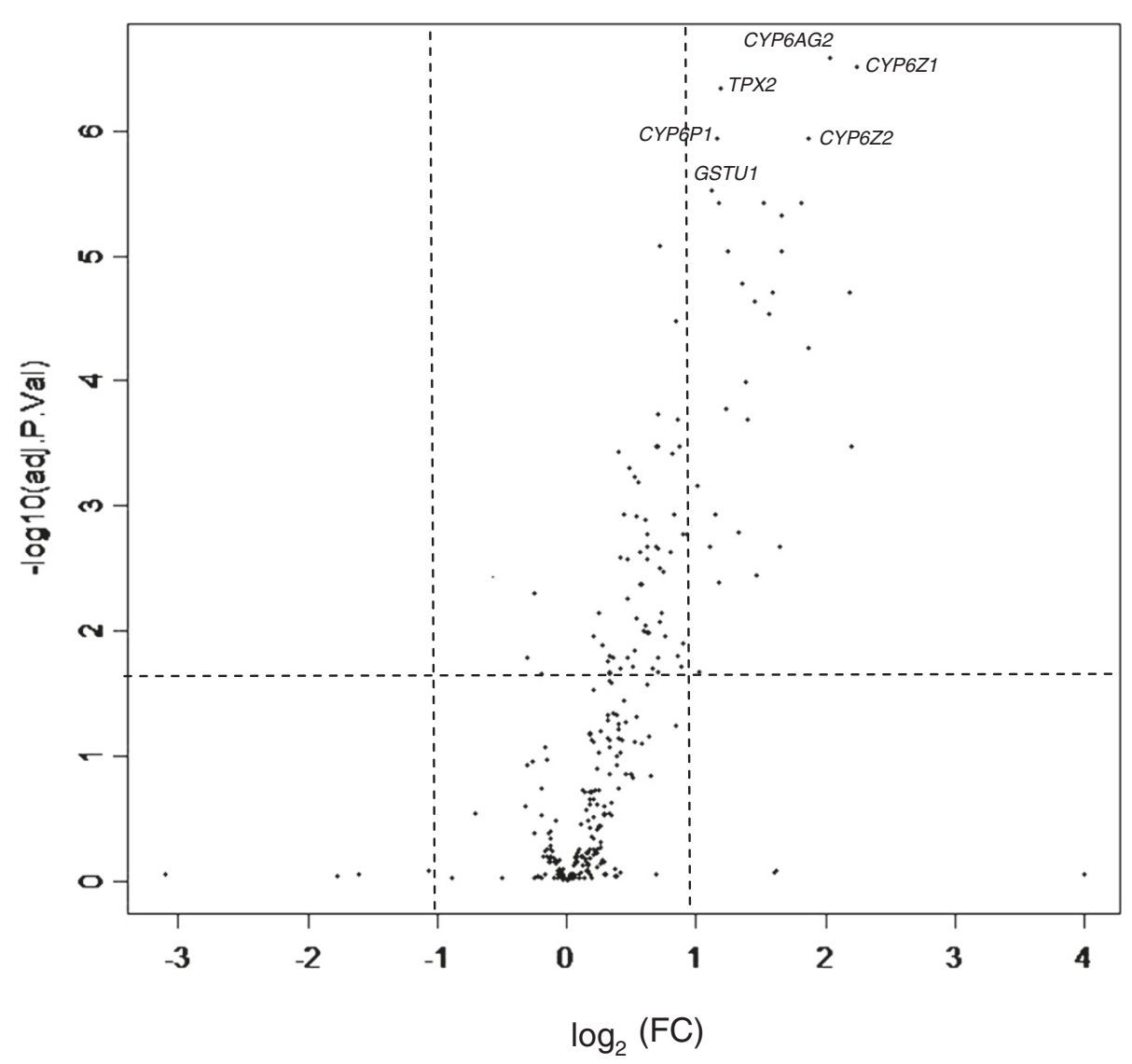

Figure 1 The volcano plot of KWAG-perm versus KWAG-base microarray data. The cut-offs for significance are indicated by the dotted lines (adj. $p$-value $\leq 0.05 ; F C \geq 2$ ) and the top 6 genes that met this criteria have been labeled. All positive FC values belong to the genes that are over-transcribed in the resistant strain (KWAG-perm), while all negative FC values belong to genes that are over-transcribed in the susceptible strain.

and pyrethroid resistant strain (MBN-DDT). These genes were mainly found to be associated with the observed cross resistance to deltamethrin (the use of synergists clarified the roles of the over-transcribed genes, and implicated $k d r$ in DDT and permethrin resistance). The gene transcripts that were identified and which are shared between the permethrin-selected (KWAG-perm) and DDT-selected strains (MBN-DDT), might explain the protection against deltamethrin in the DDT selected strain. In the process of selecting for DDT resistance it appears that there was also selection for increased transcription of genes $(n=15)$ that coincidentally protect against pyrethroids as well. However, the converse was not true and the pyrethroid selected strain did not develop DDT resistance. This implies that DDT selection pressure can also result in the de novo development of resistance to pyrethroids. Very few reports of DDT resistance without resistance to pyrethroids are available (e.g. [38]), which supports this hypothesis.

It is possible that the genes that are unique in each instance (KWAG-perm versus MBN-DDT) are more important for resistance to either type I (e.g. GSTD2) or type II pyrethroid (CYPZ1), while those that are common are likely to play a role in resistance to both kinds of pyrethroid (e.g. CYP6M2). Those genes that are shared between the pyrethroids and DDT selected strain might be able to metabolise both classes of insecticides (DDT as well as pyrethroids). This is specifically true for CYP6M2 [39], but to our knowledge similar metabolic studies are not available for the other 10 genes. The role of genes conferring carbamate resistance identified in the DDT-selected strain still needs to be clarified [8]. However, P450s have been shown to provide protection against both pyrethroids and carbamates in An. funestus [40].

\section{Conclusions}

The challenge with detoxification based resistant mechanisms is that it is not possible to develop a single molecular assay for the detection of resistance (as in the case of target-site mediated resistance, for example, $k d r$ detection). Furthermore, in target-site resistance mechanisms, genotype usually correlates with phenotypic out- 


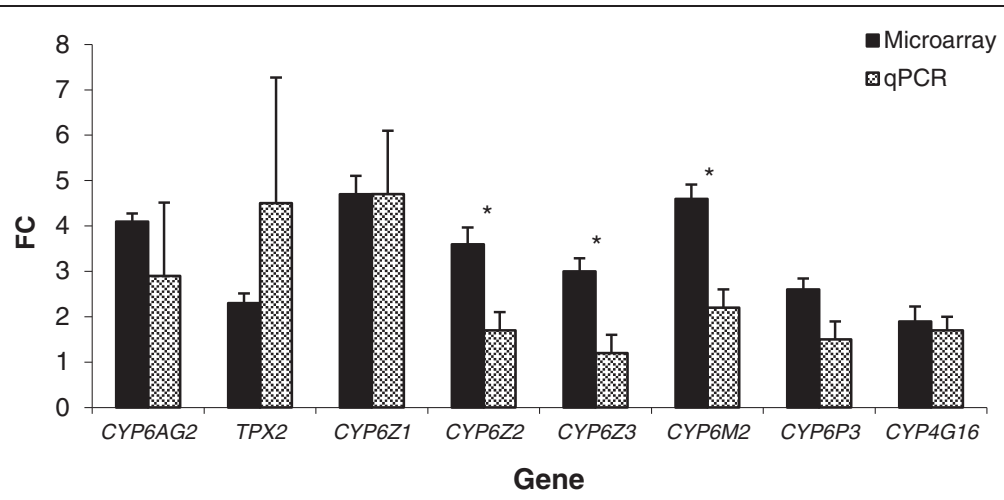

Figure 2 A comparison between average fold change (FC) +SEM values recorded using microarrays and qPCR. QPCR FC values included here for CYP6Z1, CYP6Z2, CYP6Z3, CYP6M2, CYP6P3 and CYP4G16 are those reported by Munhenga and Koekemoer [22]. Microarray and GPCR FC values were compared to each other by means of a $t$-test. Pairs of bars marked with a * are significantly different.

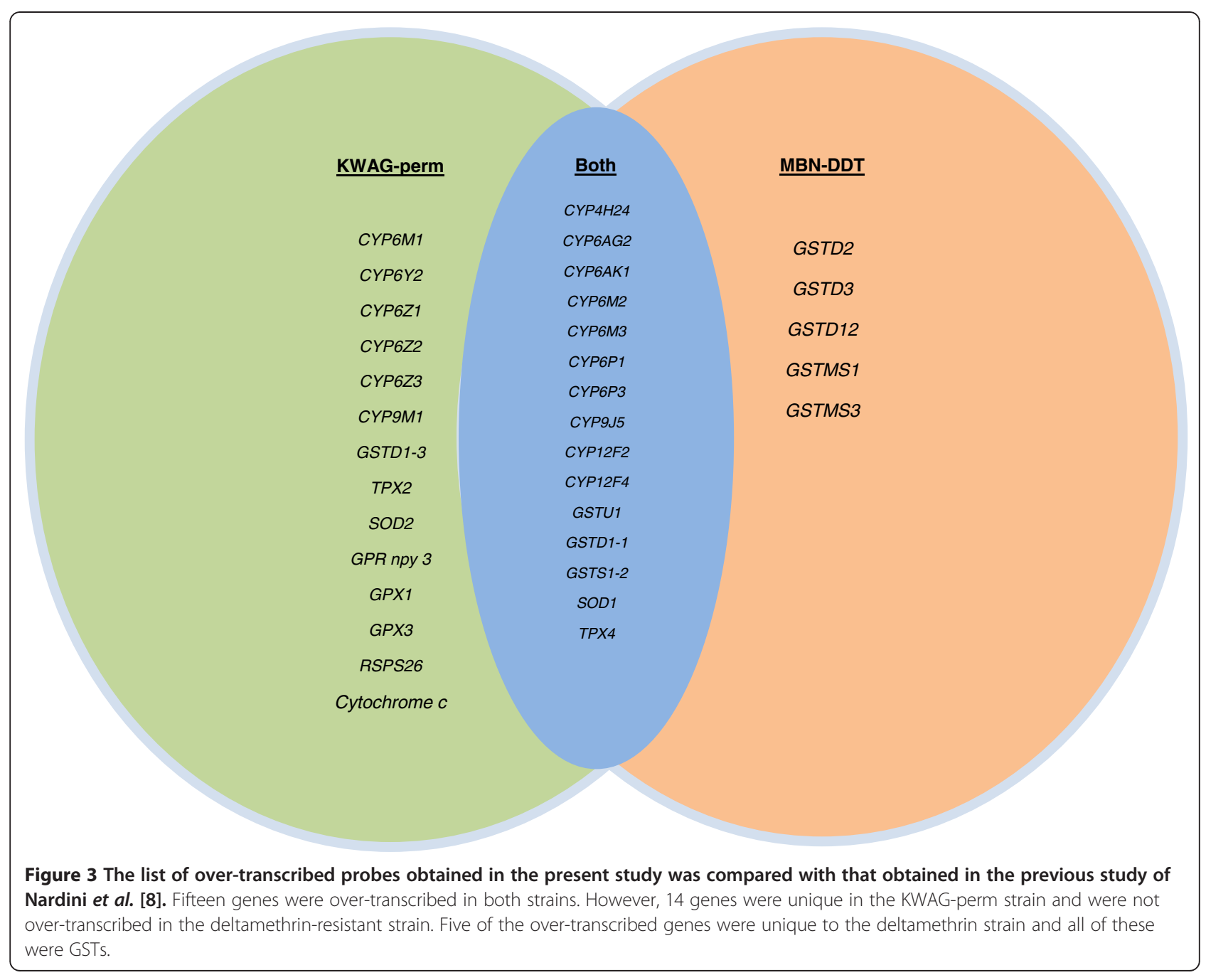


come. In the case of enzymatic resistance, this is not necessarily true. For example, three GSTs were over-transcribed in the strain here (the activity of GSTs is known to confer DDT resistance in mosquitoes $[41,42]$ ), but no DDT resistance is present in KWAG-perm. In addition, different genes were over-transcribed in the permethrin and deltamethrin resistant strains.

Microarrays are extremely useful for identifying a large number of genes that are associated with a particular resistance phenotype and in particular, transcripts that are unique to a particular phenotype. However, this technology is extremely expensive and not available in most African countries and an alternative to this approach is needed. Due to this problem, the results from the costly microarray approach used in this study were compared against those obtained by a less expensive qPCR study using the same $A n$. arabiensis strain [22]. Selection of genes to be included in this qPCR study were identified through published literature $[7,9,10,14,34]$. This approach was used successfully by Munhenga and Koekemoer [22] who found $\geq 1.5 \mathrm{FC}$ in transcription in four of the six genes that were selected for analysis. Five of the six genes from Munhenga and Koekemoer [22] were also identified through this microarray study. However, CYP6AG2 and TPX2 were novel in this study.

These data suggest that relatively inexpensive qPCR studies can successfully be used to identify increased transcription of specific metabolic genes, especially in countries that do not have access to advanced molecular systems eg. microarray analysis. However, many transcripts might be overlooked using QPCR due to the specificity of the technique. Microarray studies will in future expand the number of transcripts available for qPCR screening.

The GPIRM has initiated a "five pillar" approach against insecticide resistance. One of these pillars is to gain knowledge on mechanisms of insecticide resistance and on the effects of current insecticide resistance management strategies [1]. The present study and similar studies, contribute to achieving this goal.

\section{Abbreviations}

CYP: Cytochrome P450; DDT: Dichlorodiphenyltrichloroethane;

GPX: Glutathione peroxidase; GST: Glutathione S-transferase; kdr: Knockdown resistance; KWAG-perm: Permethrin-resistant An. arabiensis from South Africa; KZN: KwaZulu-Natal; LIMMA: Linear Models in Microarray Analysis; MBNDDT: DDT and pyrethroid resistant An. arabiensis from South Africa; qPCR: Quantitative real-time PCR; ROS: Reactive oxygen species; SOD: Superoxide dismutase; TPX: Thioredoxin peroxidase; WHO: World Health Organization.

\section{Competing interests}

The authors declare that they have no competing interests.

\section{Authors' contributions}

LN performed all the experimental work, analyses and drafted the MS. RC provided technical support for the microarray work and reviewed the MS. NC assisted with the microarray data analysis and reviewed the MS. LK conceived the project, provided funding and reviewed the MS. All authors read and approved the final manuscript.

\section{Acknowledgements}

The authors acknowledge the following funding institutions: National Research Foundation Scarce Skills Scholarships and DAAD (LN), National Research Foundation and the National Health Laboratory Service-Research Trust to LLK, partial funding from the Department of Science and Technology, National Research Foundation Research Chair Initiative grant to Maureen Coetzee. The authors also acknowledge Maureen Coetzee and Basil Brooke for comments on the manuscript. The grant holders acknowledge that opinions, findings and conclusions or recommendations expressed in any publication generated by the NRF supported research are that of the author(s), and that the NRF accepts no liability whatsoever in this regard.

\section{Author details}

'Division of the National Health Laboratory Services, Vector Control Reference Laboratory, Centre for Opportunistic, Tropical and Hospital Infections, National Institute for Communicable Diseases, Private Bag X4, Johannesburg 2131 Sandringham, South Africa. ${ }^{2}$ WITS Research Institute for Malaria, School of Pathology, Faculty of Health Sciences, University of the Witwatersrand, Johannesburg, South Africa. ${ }^{3}$ Bioinformatics and Computational Biology Unit, Department of Biochemistry, University of Pretoria, Pretoria, South Africa.

Received: 10 June 2013 Accepted: 2 August 2013

Published: 8 August 2013

\section{References}

1. World Health Organization: Global Plan for Insecticide Resistance Management in Malaria Vectors; 2012.

2. Coosemans M, Carnevale P: Malaria vector control: a critical review on chemical methods and insecticides. Ann Soc Belg Med Trop 1995, 75:13-31.

3. Liu N, Xu Q, Zhu F, Zhang L: Pyrethroid resistance in mosquitoes. Insect Sci 2006, 13:159-166.

4. Hemingway J, Hawkes NJ, McCarroll L, Ranson H: The molecular basis of insecticide resistance in mosquitoes. Insect Biochem Mol Biol 2004, 34:653-665

5. Bass C, Nikou D, Donnelly MJ, Williamson MS, Ranson H, Ball A, Vontas J, Field LM: Detection of knockdown resistance $(k d r)$ mutations in Anopheles gambiae: a comparison of two new high-throughput assays with existing methods. Malaria J 2007, 6:111.

6. Brooke BD, Koekemoer LL: Major effect genes or loose confederations? The development of insecticide resistance in the malaria vector Anopheles gambiae. Parasit Vectors 2010, 3:74.

7. David JP, Strode C, Vontas J, Nikou D, Vaughan A, Pignatelli PM, Louis C, Hemingway J, Ranson H: The Anopheles gambiae detoxification chip: a highly specific microarray to study metabolic-based insecticide resistance in malaria vectors. Proc Natl Acad Sci USA 2005, 102:4080-4084

8. Nardini L, Christian RN, Coetzer N, Ranson H, Coetzee M, Koekemoer LL: Detoxification enzymes associated with insecticide resistance in laboratory strains of Anopheles arabiensis of different geographic origin. Parasit Vectors 2012, 5:113.

9. Müller P, Donnelly MJ, Ranson H: Transcription profiling of a recently colonised pyrethroid resistant Anopheles gambiae strain from Ghana. BMC Genom 2007, 8:36.

10. Müller P, Chouaïbou M, Pignatelli P, Etang J, Walker ED, Donnelly MJ, Simard $\mathrm{F}$, Ranson $\mathrm{H}$ : Pyrethroid tolerance is associated with elevated expression of antioxidants and agricultural practice in Anopheles arabiensis sampled from an area of cotton fields in Northern Cameroon. Mol Ecol 2007, 17:1145-1155.

11. Vontas J, David JP, Nikou D, Hemingway J, Christophides GK, Louis C, Ranson H: Transcriptional analysis of insecticide resistance in Anopheles stephensi using cross-species microarray hybridization. Insect Mol Biol 2007, 16:315-324.

12. Müller $P$, Warr E, Stevenson BJ, Pignatelli PM, Morgan JC, Steven A, Yawson AE, Mitchell SN, Ranson H, Hemingway J, et al: Field-caught permethrin-resistant Anopheles gambiae overexpress CYP6P3, a P450 that metabolises pyrethroids. PLoS Genet 2008, 4:e1000286.

13. Christian RN, Strode C, Ranson H, Coetzer N, Coetzee M, Koekemoer LL: Microarray analysis of a pyrethroid resistant African malaria vector, Anopheles funestus, from Southern Africa. Pestic Biochem Physiol 2011, 99:140-147. 
14. Nikou D, Ranson H, Hemingway J: An adult-specific CYP6 P450 gene is overexpressed in a pyrethroid-resistant strain of the malaria vector Anopheles gambiae. Gene 2003, 318:91-102

15. Ranson H, Paton MG, Jensen B, McCarroll L, Vaughan A, Hogan JR, Hemingway J, Collins FH: Genetic mapping of genes conferring permethrin resistance in the malaria vector, Anopheles gambiae. Insect Mol Biol 2004, 13:379-386.

16. Mouatcho JC, Munhenga G, Hargreaves K, Brooke BD, Coetzee M, Koekemoer LL: Pyrethroid resistance in a major African malaria vector Anopheles arabiensis from Mamfene, northern KwaZulu-Natal, South Africa. S Afr J Sci 2009, 105:127-131.

17. Coetzee M, Koekemoer LL: Molecular systematics and insecticide resistance in the major African malaria vector Anopheles funestus. Annu Rev Entomol 2013, 58:393-412.

18. Coetzee M, Craig M, le Sueur D: Distribution of African malaria mosquitoes belonging to the Anopheles gambiae complex. Parasitol Today 2000, 16:74-77.

19. Maharaj R, Mthembu DJ, Sharp BL: Impact of DDT re-introduction on malaria transmission in KwaZulu-Natal. S Afr Med J 2005, 95:871-874.

20. Gericke A, Govere JM, Durrheim DN: Insecticide susceptibility in the South African malaria mosquito Anopheles arabiensis (Diptera: Culicidae). S Afr J Sci 2002, 98:205-208.

21. Hargreaves K, Hunt RH, Brooke BD, Mthembu J, Weeto MM, Awolola TS, Coetzee M: Anopheles arabiensis and An. quadriannulatus resistance to DDT in South Africa. Med Vet Entomol 2003, 17:417-422.

22. Munhenga G, Keoekemoer LL: Differential Expression of cytochrome P450 genes in a laboratory selected Anopheles arabiensis colony. Afr J Biotech 2011, 10:12711-12716

23. Smyth GK: Limma: linear models for microarry data. New York: Springer; 2005.

24. Ritchie ME, Silver J, Oshlack A, Holmes M, Diyagama D, Holloway A, Smyth GK: A comparison of background correction methods for two-colour microarrays. Bioinformatics 2007, 23:2700-2707.

25. van Heerden J, Walford S-A, Shen A, Illing N: A framework for informed normalization of printed microarrays. S Afr J Sci 2007, 103:381-390.

26. Smyth GK, Speed TP: Normalization of cDNA microarray data. Methods 2003, 31:265-273.

27. Pfaffl MW: A new mathematical model for relative quantification in real-time RT-PCR. Nucleic Acids Res 2001, 29:e45.

28. Martins AJ, Ribeiro CD, Bellinato DF, Peixoto AA, Valle D, Lima JB: Effect of insecticide resistance on development, longevity and reproduction of field or laboratory selected Aedes aegypti populations. PLoS One 2012, 7:e31889.

29. Scott JG: Cytochromes P450 and insecticide resistance. Insect Biochem Mol Biol 1999, 29:757-777.

30. Vontas JG, Small GJ, Hemingway J: Glutathione S-transferases as antioxidant defence agents confer pyrethroid resistance in Nilaparvata lugens. Biochem J 2001, 357:65-72.

31. Bauer H, Kanzok SM, Schirmer RH: Thioredoxin-2 but not thioredoxin-1 is a substrate of thioredoxin peroxidase-1 from Drosophila melanogaster: isolation and characterization of a second thioredoxin in D. Melanogaster and evidence for distinct biological functions of Trx-1 and Trx-2. J Biol Chem 2002, 277:17457-17463.

32. James RR, $\mathrm{Xu} \mathrm{J}$ : Mechanisms by which pesticides affect insect immunity. J Invertebr Pathol 2012, 109:175-182.

33. Fridovich I: The biology of oxygen radicals. Science 1978, 201:875-880.

34. Djouaka RF, Bakare AA, Coulibaly ON, Akogbeto MC, Ranson H, Hemingway J, Strode C: Expression of the cytochrome P450s, CYP6P3 and CYP6M2 are significantly elevated in multiple pyrethroid resistant populations of Anopheles gambiae s.s. from Southern Benin and Nigeria. BMC Genom 2008, 9:538.

35. Hemingway J, Dunbar SJ, Monro AG, Small GJ: Pyrethroid resistance in German cockroaches (Dictyoptera: Blattelidae): resistance levels and underlying mechanisms. J Econ Entomol 1993, 86:1631-1638.

36. Lagadic L, Cuany A, Bergé JB, Echaubard M: Purification and partial characterization of glutathione S-transferases from insecticide-resistant and lindane-induced susceptible Spodoptera littoralis (Boisd.) larvae. Insect Biochem Mol Biol 1993, 23:467-474.

37. Kostaropoulos I, Papadopoulos Al, Metaxakis A, Boukouvala E, Papadopoulou-Mourkidou E: Glutathione S-transferase in the defence against pyrethroids in insects. Insect Biochem Mol Biol 2001, 31:313-319.
38. Namountougou M, Simard F, Baldet T, Diabate A, Ouedraogo JB, Martin T, Dabire RK: Multiple insecticide resistance in Anopheles gambiae s.l. populations from Burkina Faso, West Africa. PLoS One 2012, 7:e48412.

39. Mitchell SN, Stevenson BJ, Muller P, Wilding CS, Egyir-Yawson A, Field SG, Hemingway J, Paine MJ, Ranson H, Donnelly MJ: Identification and validation of a gene causing cross-resistance between insecticide classes in Anopheles gambiae from Ghana. Proc Natl Acad Sci USA 2012, 109:6147-6152.

40. Kloke RG, Nhamahanga E, Hunt RH, Coetzee M: Vectorial status and insecticide resistance of Anopheles funestus from a sugar estate in southern Mozambique. Parasit Vectors 2011, 4:16.

41. LA Prapanthadara HJ, Ketterman AJ: Partial purification and characterisation of glutathione s-transferases involved in DDT resistance from the mosquito Anopheles gambiae. Pest Biochem Physiol 1993, 47:119-133.

42. DE Grant DF, Hammock BD: Glutathione-S-transferase isozymes in Aedes aegypti - purification, characterization, and isozyme-specific regulation. Insect Biochem 1991, 21:421-433.

doi:10.1186/1756-3305-6-229

Cite this article as: Nardini et al.: DDT and pyrethroid resistance in

Anopheles arabiensis from South Africa. Parasites \& Vectors 2013 6:229.

\section{Submit your next manuscript to BioMed Central and take full advantage of:}

- Convenient online submission

- Thorough peer review

- No space constraints or color figure charges

- Immediate publication on acceptance

- Inclusion in PubMed, CAS, Scopus and Google Scholar

- Research which is freely available for redistribution

Submit your manuscript at www.biomedcentral.com/submit
C Biomed Central 